\title{
A systematic review of remote otological assessment using video-otoscopy over the past 10 years: reliability and applications
}

\author{
Christopher Metcalfe ${ }^{1}\left[\right.$ ] Jameel Muzaffar ${ }^{1}\left[\right.$ Linda Orr $^{1} \cdot$ Christopher Coulson $^{1}(\mathbb{C}$
}

Received: 10 August 2020 / Accepted: 24 December 2020 / Published online: 24 January 2021

(c) The Author(s), under exclusive licence to Springer-Verlag GmbH, DE part of Springer Nature 2021

\begin{abstract}
Purpose This systematic review describes and evaluates the current literature on remote otological assessment using videootoscopy with regards to reliability and potential applications.

Methods Systematic review was conducted in accordance with the PRISMA statement. There was heterogeneity of included studies, so a descriptive analysis was undertaken.

Results Seventeen studies were included for analysis conducted across a variety of healthcare settings. Overall, there was a trend towards acquisition of adequate images for diagnosis by non-otolaryngology-specialist facilitators with reasonable agreement between asynchronous images and controls; however, there was significant variation between the studies.

Conclusion Remote otological assessment using video-otoscopy shows potential as a safe and effective method for detecting the presence of ear disease in a wide range of healthcare settings. Barriers to the acquisition of adequate images include the presence of obstructing cerumen, and strategies to deal with this should be considered. Further work is required to evaluate endoscope-based systems to assess whether they will allow the acquisition of higher quality images.
\end{abstract}

Keywords Video-otoscopy $\cdot$ Tele-otoscopy $\cdot$ Tele-otology $\cdot$ Telehealth $\cdot$ Smartphone otoscopy $\cdot$ Technology

\section{Introduction}

Ear disease and hearing loss contribute significantly to population disease burdens, with approximately 460 million people worldwide living with disabling hearing loss [1]. Such disease is often preventable or easily treated when a diagnosis is made. Complete assessment of patients with ear disease requires direct visualisation of the ear canal, tympanic membrane and middle ear structures to make an accurate diagnosis and instigate appropriate treatment, and this is carried out as part of a face-to-face examination using either an otoscope, otoendoscope or a microscope. In recent years, technological advances have seen the genesis of devices which can be used to capture images of the ear and facilitate remote diagnosis. The potential applications for this technology are numerous. Generally, the main focus of video-otoscopy has been on its ability to assess remote populations, where access to specialist otolaryngology

Christopher Metcalfe

christophermetcalfe@nhs.net

1 Department of Otolaryngology, Queen Elizabeth Hospital Birmingham, Edgbaston, Birmingham B15 2TH, UK services is sparse. Remote otoscopic assessment has also been used to screen for ear disease in large populations with a high prevalence of ear disease, where poor or developing healthcare infrastructure limits access to otolaryngology services $[2,3]$. Remote assessment may also have a place in more established healthcare systems such as the UK's National Health Service (NHS), where otolaryngology referrals make up nearly $8 \%$ of all referrals from primary to secondary care [4]. The introduction of remote assessment may facilitate better triage of referrals and ensure patients are referred to the right specialist in an appropriate timescale. Or, if pushed to its limit, entirely remote diagnosis as the norm, with face-to-face consultations only for those who cannot be managed remotely. The emergence of a novel coronavirus, SARS-CoV-2, in late 2019 and the subsequent global pandemic of COVID19 disease has also shone a light on the value of remote assessment, facilitating the delivery of healthcare whilst shielding vulnerable patients from the hospital environment. Finally, secure storage and archiving of images would form an integral component of patients' medical records, providing accurate historical documentation of clinical findings and promoting better communication between healthcare professionals. This systematic review 
describes and evaluates the current literature on remote assessment using video-otoscopy with regards to reliability and potential applications.

\section{Methods}

\section{Study design}

A systematic review of published data pertaining to the use of video-otoscopy for remote otological assessment was conducted in accordance with the PRISMA statement [5].

\section{Search strategy}

Electronic searches of PubMed and the Cochrane Database were performed, with no limits placed on language. Due to advances in technology, it was the authors' consensus to limit searches to the last 10 years (2009-2019). Searches were performed using the following terms: (hearing OR otology OR otolaryngology OR otorhinolaryngology OR ENT) AND (Tele OR remote OR video-otoscopy).

\section{Study selection}

All abstracts were assessed for eligibility against the inclusion criteria (Table 1) by the two authors (anonymised for peer review). Where ambiguities existed, the full paper was obtained. Reference lists from selected studies were assessed against the inclusion criteria. Any disagreements were discussed with the senior author.

\section{Data extraction and analysis}

Data was collected on eight domains (Table 2) and entered into a spreadsheet for analysis. Initial data extraction was performed by a single author (anonymised for peer review) and this was verified by a second author (anonymised for peer review) for quality assurance. There was heterogeneity of included studies both in methodology and reported outcomes, so a descriptive analysis was undertaken. Data extraction was performed using Microsoft Excel (Microsoft, WA, USA).

\section{Results}

\section{Summary}

Initial searches returned 588 abstracts, of which 26 full papers were retrieved. Nine of these were excluded, leaving 17 papers for analysis. Number of patients varied

Table 2 Data collected

\begin{tabular}{l}
\hline Data collected \\
\hline Author/title \\
Methodology \\
Patient population \\
Setting \\
Who performed video otoscopic \\
assessment? \\
Who assessed the remote \\
images? \\
Device used \\
Reported outcomes
\end{tabular}

Table 1 Inclusion/exclusion criteria

\begin{tabular}{l}
\hline Inclusion criteria \\
\hline Studies with any methodology other than single-case reports \\
Studies examining the use of remote assessment with video/photo-otoscopy (via any equipment) for any \\
ear and/or hearing problem \\
Synchronous or asynchronous assessment \\
Human studies \\
Adults and children \\
Any language of publication \\
Any geographical region \\
Year of publication 2009-2019 \\
Exclusion criteria \\
Non-human/simulated studies \\
Literature reviews \\
Studies using video-otoscopy without remote assessment \\
Studies not reporting on quality of images, ability to make diagnosis or diagnostic concordance
\end{tabular}


considerably between studies with a total of 4266 patients included (range 3-3000). In addition to this, 1 study included an analysis of 210 still images of tympanic membranes collected prospectively from an unspecified number of adult patients. Six studies included adults only [6-11], seven studies included children only [12-18], three studies included both adults and children [2, 3, 19] and one study did not specify population age [20]. A range of devices were used to perform video-otoscopy (Table 3 ). In seven studies $[2,6,12-14,18,19]$, non-medical healthcare facilitators performed the remote assessment; however, in two studies, the parents of paediatric patients were used [16, 17]. All but two studies $[10,11]$ utilised an asynchronous assessment of remote images.

\section{Quality of images}

There was heterogenous reporting of image quality. Overall, there was a trend towards acquisition of adequate images for diagnosis by non-otolaryngology-specialist facilitators, with a pooled failure rate of $26 \%$; however, there was significant variation between studies (range 7-81.9\%). Exclusion of the study with a failure rate of $81.9 \%$ [15] improves the pooled failure rate to $19 \%$ (range 7-35.7\%). The otoscopic images assessed by Moberly et al. were all acquired by a fellowshiptrained neurotologist and poor-quality images (e.g. fuzzy or poor lighting) $(80 / 300,27 \%)$ were excluded from their analysis. In the majority of studies, acquisition of adequate images was either based on ability to make a diagnosis or not specified; however, in the remainder of studies, various scales were used as a measure of image quality. Two studies $[6,14]$ used a scale of $0-2$ (corresponding to unacceptable, acceptable, excellent) with reference to image focus, light, obscuring objects, and composition. Images acquired by a facilitator were rated as either acceptable or excellent in $75.4 \%$ [6] and 87\% [14] of cases, respectively. Demant et al. [15] used a 5 point Likert scale to classify the videos as either useful (rated as "agree" or "strongly agree") or not useful (rated as neither agree nor disagree, disagree, or strongly disagree); however, the mean proportion of useful videos was only $18.1 \%$. Wu et al. [20] used a scale of good, fair or poor to assess images. Image quality varied between their three patients with a rating of 'good' for a patient with a tympanic membrane perforation, fair for an acute otitis media and poor for atrophic scarring of the tympanic membrane. There was $100 \%$ concordance between the three assessors. The presence of cerumen was an issue noted in most studies [2, 3, 7, 12, 14-19]. Cerumen removal was deemed necessary to make an assessment in between 9 and $36.6 \%$ of ears, and the presence of cerumen precluded a diagnosis or assessment of the tympanic membrane in $8-31.3 \%$ of ears.

\section{Agreement between asynchronous images and controls}

Eight studies [3, 6, 8, 11-14, 17] used a 'gold standard' clinical assessment as a control with which to compare their remote assessment using video-otoscopy; four of these used on site microscopy by an otolaryngologist, three used on site otoscopy by an otolaryngologist and one used a face-to-face assessment by an otolaryngologist but did not specify what this involved [11]. Six studies [3, 6, 12-14, 17] presented a quantification of diagnostic concordance between the remote asynchronous assessment and the gold standard using kappa coefficients (Table 4). Moberly et al. observed that average concordance between asynchronous image assessment and control was $72 \%$ for normal tympanic membranes, however, it ranged from 48 to $100 \%$ for different types of ear pathology. Agreement between the 12 assessors was low across the various diagnoses $(k=0.34)$. Yulzari et al. utilised a system of synchronous assessment in which the otolaryngologist
Table 3 Devices used for videootoscopy

\begin{tabular}{ll}
\hline Study & Device used \\
\hline Biagio 2014, Lundberg 2014, Lundberg 2017 & Dino-Lite Pro Earscope \\
Demant 2019, Mandavia 2018 & Cupris ${ }^{\circledR}$ \\
Damery 2019, Sebothoma 2018 & AURICAL ${ }^{\circledR}$ OTO-cam 300 (Otometrics) \\
Gupta 2017, Mandavia 2018 & ENTraview device (Medtronic) \\
Erkkola-Anttinen 2019, Shah 2018 & CellScope Oto/iPhone \\
Biagio 2013 & Welch Allyn digital MacroView videootoscope \\
Seim 2018 & Storz otoscope with Quintree software \\
Wu 2014 & Prototype smartphone adapter with endoscope* \\
Yancey 2019 & HearScope ${ }^{\text {TM }}$ \\
Yulzari 2018 & Clearscope smartphone endoscope adapter* \\
Moberly 2018 & JEDMED Horus + HD Video Otoscope (St \\
& Louis, Missouri, USA) \\
\hline
\end{tabular}

*Denotes devices that are endoscope-based rather than otoscope-based 
Table 4 A summary of agreement between remote assessment and control expressed as Cohen's kappa coefficients

\begin{tabular}{llll}
\hline Study & Control & Remote assessment & Diagnostic agreement \\
\hline $\begin{array}{l}\text { Biagio, 2013 } \\
\text { Biagio, 2014 }\end{array}$ & Microscopy by otolaryngologist & Healthcare facilitator & $k=0.596$ \\
& Microscopy by otolaryngologist & Healthcare facilitator & $k=0.70(4 \text { weeks })^{*}$ \\
Lundberg, 2014 & Microscopy by otolaryngologist & Healthcare facilitator & $k=0.75(8 \text { weeks })^{*}$ \\
& & & $k=0.73(4 \text { weeks })^{*}$ \\
Lundberg, 2017 & Microscopy by otolaryngologist & Healthcare facilitator & $k=0.77(8 \text { weeks })^{*}$ \\
Mandavia, 2018 & & & $k=0.769(4 \text { weeks })^{*}$ \\
Shah, 2018 & Otoscopy by otolaryngologist & 1 ENT trainee, 1 GP & $k=0.860(8 \text { weeks })^{*}$ \\
& Otoscopy by otolaryngologist & Parents of paediatric patients & $k=0.42$ \\
\end{tabular}

These values are generally interpreted as follows: $0.21-0.40$ fair agreement, $0.41-0.60$ moderate agreement, $0.61-0.80$ substantial agreement, and $0.81-1.00$ near perfect agreement ${ }^{25}$

*The same remotely acquired images were assessed at both 4 and 8 weeks

Table 5 Sensitivity, specificity, PPV and NPV for remote assessment using video-otoscopy

\begin{tabular}{lllll}
\hline Study & Sensitivity & Specificity & PPV & NPV \\
\hline Biagio, 2013 & 0.85 & 0.89 & $\mathrm{x}$ & $\mathrm{x}$ \\
Biagio, 2014 & 0.78 & 0.95 & 0.74 & 0.96 \\
Lundberg, 2014 & 0.68 & 0.98 & 0.78 & 0.96 \\
Lundberg, 2017 & 0.79 & 0.96 & 0.82 & 0.96 \\
Mandavia, 2018 & 0.94 & 0.96 & 0.91 & 0.97 \\
Pooled mean & 0.81 & 0.95 & 0.81 & 0.96 \\
\hline
\end{tabular}

reviewed a live stream of remotely acquired images and was, therefore, able to direct the acquisition of images. Comparison of remote diagnoses and face-to-face diagnoses by an otolaryngologist demonstrated a concordance of $86.4 \%$. Five studies [3, 6, 12-14] calculated sensitivity, specificity, positive predictive values (PPV) and negative predictive values (NPV) for diagnosis using remote video-otoscopy versus control (Table 5).

\section{Quality of studies}

The majority of studies were observational studies, however, one study [16] added an experimental element by investigating the effects of educational intervention on the quality of acquired images. Sample size was variable across studies with the majority specifying how many ears were assessed as a true sample size in addition to the number of patients. Wu et al. [20] only included three otology patients in their feasibility study. Quality of acquired images was commonly reported; however, there was variation in the measures used with most presenting subjective comments on 'usefulness' or 'acceptability'. Likewise, diagnostic concordance between remote assessment and a control was used in only eight studies $[3,6,8,11-14,17]$ with variation in the control, including microscopy and otoscopy, usually by a single otolaryngology specialist. The study by Demant et al. [15] stood out as the video-otoscopy failure rate was notably higher than other studies. Three of the included studies [12-14] were conducted at the Witkoppen Health and Welfare Clinic in South Africa by the same researchers and included the same number of patients with the same age-range. Each study had a different design and different outcomes reported; however, it is possible that they were all conducted using the same population of patients. The study by Moberly et al. was unique as all images for remote assessment were acquired by a fellowship-trained neurotologist and rated by 12 different neurotologists. Finally, it should be noted that image acquisition is technology dependent and there was variation in the devices used (Table 3).

\section{Discussion}

This systematic review describes and evaluates the last 10 years of the literature on remote assessment using videootoscopy, with regards to reliability and potential applications. As far as the authors are aware, this is the first systematic review on this topic. Outcomes from the 17 included studies provide more information about devices currently being employed and how effective they are at acquiring useful images. We also discuss the potential applications of this technology and suggest where future research should be directed.

\section{Image quality}

There was a general trend towards acquisition of adequate images across all studies other than Demant et al. who 
reported only $18.1 \%$ of images as useful, despite the Cupris ${ }^{\circledR}$ device they utilised proving very effective when used in the study by Mandavia et al. The main reason for this was suboptimal insertion of the otoscope which may have reflected facilitator inexperience, compounded by an exclusively paediatric patient population which may have made otoscopy more challenging. Furthermore, they aimed to replicate 'real-world' conditions by electing not to perform cerumen removal which contributed to failure in a number of ears. Adequate images were acquired in $81 \%$ of ears across the remaining studies when otoscopy was performed by nonotolaryngology-specialist facilitators, which suggests that current devices are a feasible option for remote otological assessment. This also demonstrates potential for reliable remote assessment by trained facilitators, where otolaryngology specialists are involved only in the interpretation of acquired images. It is notable that Moberly et al. used a fellowship-trained otologist to acquire all images which makes it challenging to compare their results to the studies using non-otolaryngology-specialist facilitators. Despite this, $27 \%$ (80/300) of images were excluded from analysis due to poor quality (e.g. fuzzy image) or poor lighting, which is perhaps surprising as the use of a fellowship-trained otologist should maximise the potential of a higher quality image being obtained. One could postulate that this is, therefore, a reflection of other factors such as the device used or the patient's anatomy, rather than facilitator skill. A clear barrier to remote otoscopic assessment is the presence of cerumen obscuring the tympanic membrane. Indeed, cerumen removal was required in up to $36.6 \%$ of ears. It should also be noted that some studies excluded ears from their analysis where the presence of cerumen precluded a diagnosis, and therefore, the quoted success of remote assessment is likely to be exaggerated compared to a real-world situation where cerumen will often obscure the tympanic membrane. There may be solutions to this, for example, using a short course of cerumenolytic ear drops and bringing the patient back for further review, despite evidence for the efficacy of cerumenolytics being limited [21]. There may also be a role for ear syringing in certain situations [22], as this technique is noninvasive and does not require specialist equipment. Whilst it is relatively low risk, there is a vogue away from syringing due to the risk of otitis externa and its contraindication in those with a perforation, discharge or grommets. In situations where cerumen removal is not possible, formal referral to a facility with specialist equipment would be necessary. When discussing the quality of images, it is interesting to note that the majority of studies used a video-otoscopebased system rather than an endoscope-based system. In the context of trained facilitators conducting unsupervised otoscopy, it could be argued that using an otoscope rather than a rigid endoscope is a safer technique as it is unlikely that the speculum would traumatise the tympanic membrane, for example, in the case of a non-cooperative paediatric patient. Conversely, in the hands of an inexperienced operator, the potential for trauma to the ear canal, tympanic membrane and potentially even the ossicles, is greater with use of a rigid endoscope. Anecdotally, there may also be a case that otoscopes are slightly more robust than their more delicate endoscope counterparts, which is of potential relevance when used to assess remote and rural populations. However, when discussing image quality in isolation, endoscopes have numerous advantages over otoscopes, including a wider field of view, higher resolution and better magnification which should lead to the acquisition of higher quality images. Two of the included studies used an endoscope-based system; however, there is limited evidence from these to suggest any advantage of the endoscope in terms of image quality. Yulzari et al. [11] utilised synchronous assessment in which images were live-streamed to a remote assessor, and therefore, the remote physician was able to direct the examination to aid acquisition of optimal images. They demonstrated high concordance $(86.4 \%)$ between remote diagnosis and control. Wu et al. [20] only included three otology patients so the power of their study is limited with regards to the use of an endoscope. Regardless of the device used to acquire images, it is clear that in real-world scenarios, there must be an escalation plan in cases of inadequate image acquisition which includes either managing the cause of poor images (e.g. cerumen removal), repeat assessment or face-to-face review with an otolaryngologist. This will ensure that serious pathology is not missed and that patients are managed appropriately. As with any situation in which new technology is introduced, it is vital that practice is audited to maintain patient safety.

\section{Diagnostic concordance for remote assessment versus controls}

Comparison of remote assessment with video-otoscopy compared to an accepted 'gold-standard' method of clinical assessment is imperative when assessing its validity. Eight studies utilised a control with which to compare remote assessment and investigate agreement between the two diagnoses. Generally, agreement was very high between the two methods of assessment which suggests that the images acquired by video-otoscopy are sufficient to make an accurate diagnosis. This was fairly consistent across studies using different devices. Likewise, where studies presented sensitivities, specificities, PPVs and NPVs for remote assessment versus control, video-otoscopy performed well. It is likely that concordance will vary depending not just on the quality of image obtained, but also the type of pathology being assessed. This was well demonstrated by Moberly et al. [8] who noted a mean concordance of over $70 \%$ for normal tympanic membranes; however, there was significant variation 
where pathologies were involved. This ranged from a mean concordance of $100 \%$ for obvious findings, such as the presence of a ventilation tube, down to less than $50 \%$ for a perforation. It must be emphasised that the conditions of this study were favourable, as images were obtained and assessed by fellowship-trained otologists. The accuracy of assessment may be improved using adjuvant tests such as puretone audiometry and tympanography; however, this requires the use of additional equipment and training for facilitators. None of the included studies looked specifically at the use of adjuvant investigations and whether this improved diagnostic concordance; however, Sebothoma et al. [9] suggested that remote assessment of video-otoscopy plus tympanometry, is superior in identifying middle ear effusions compared to tympanometry alone. It could be suggested that the ability of remote assessment to answer the binary question of whether an examination is 'normal' or 'abnormal' is more important that its ability to distinguish between specific pathologies, as this is likely to be the trigger for further assessment by an otolaryngology specialist.

\section{Training of facilitators}

Training is an important factor when non-otolaryngologyspecialists are being utilised to obtain images as there is a learning curve associated with otoscopy [23]. Seven included studies used trained facilitators to perform their remote assessment, demonstrating that this is a feasible way of obtaining adequate images. In addition to this, the parents of paediatric patients were also used in two studies; however, the results were variable. Shah et al. demonstrated low concordance when parents performed video-otoscopy $(k=0.42)$ but this improved significantly with physician-performed video-otoscopy $(k=0.71)$. Erkkola-Anttinen [16] et al. investigated the effects of educational intervention on the quality of acquired images when the parents of paediatric patients performed otoscopy. The teaching intervention was a faceto-face exercise for approximately $1 \mathrm{~h}$, including information on ear anatomy, types of pathology, example images of otoscopy and an opportunity to practice with the equipment. The teaching intervention was shown to significantly improve the technical quality of the videos, underlining the importance of training. It is also notable that no adverse events were observed during otoscopy performed by parents, suggesting that this is a safe technique. Furthermore, there is evidence that remote training packages may be effective in improving both theoretical knowledge and otoscopy skills for remote assessment [24].

\section{Potential applications}

Implementation of remote otological assessment using video-otoscopy can be applied to a variety of healthcare settings. The potential benefits are obvious when it comes to the assessment of remote populations, where access to specialist otolaryngology services is often limited or absent. The socioeconomic circumstances of isolated populations are also a factor, meaning that it may be inconvenient and expensive to travel regularly to the nearest specialist centre for assessment and follow-up. Effective acquisition of otoscopic images, either at a primary care level or by trained healthcare facilitators, offers a solution whereby patients can be assessed remotely by an otolaryngology specialist at the closest tertiary centre. Where medical treatment is required, this could be directed remotely, but this would also facilitate identification of patients who need specialist assessment or surgical intervention. Likewise, where surgical intervention has been undertaken, remote assessment may be a viable option for follow-up to reduce the number of times patients have to travel to a specialist centre postoperatively [25]. Lack of access to otolaryngology services is often compounded by a high prevalence of ear disease in some isolated populations, such as the people of Greenland [26] and Aboriginal children [27]. A high prevalence of ear disease is also seen in countries such as India [28], where a large population and a relatively poorly developed healthcare infrastructure limits access to otolaryngology services. Such circumstances present another opportunity for the utilisation of remote assessment using video-otoscopy, where implementation of outreach programs, using trained facilitators, can screen for ear disease in large populations and identify pathology that needs specialist review. This was well demonstrated by Gupta et al. [2] who screened 3000 patients, identifying that over half of them required referral for specialist otolaryngology assessment. Almost half of the patients who attended for specialist assessment were deemed to require surgical intervention. There is also scope for remote otological assessment to be of benefit to more established healthcare systems such as the NHS in the UK, where otolaryngology presentations are common and subsequent referrals from primary to secondary care make up almost $8 \%$ of all referrals [4]. As a result, waiting times can be a problem and there is pressure to optimise services to reduce waiting times [4]. Furthermore, there is often a lack of confidence regarding the assessment of otolaryngology patients by general practitioners. Training general practitioners to perform video-otoscopy for remote assessment would optimise the relationship between primary and secondary cares by facilitating prompt assessment by an otolaryngology specialist, with subsequent feedback to the primary care provider. The benefits of this are numerous, for example, the time between GP-patient consultation and specialist input would be minimised. This would also facilitate identification of patients who can be managed in the community, without waiting for outpatient review, thus appropriate treatment can be administered promptly. Likewise, 
it may identify patients who need more urgent review and facilitate fast-tracking of referrals. It could allow relevant investigations to be performed whilst the patient is awaiting an outpatient appointment and the results of these would then be available for the specialist during the face-to-face review, for example, computed tomography of the temporal bones in patients with obvious cholesteatoma identified on remote assessment. Finally, there are educational benefits whereby primary care practitioners get prompt feedback on their clinical assessment. There may also be a shift towards community-based follow-up for post-operative patients. This would be of particular value in certain subgroups such as elderly or disabled patients, in whom access to an otolaryngology centre may be more inconvenient than their local primary care centre. The importance of this has been further highlighted by the emergence of a novel coronavirus, SARSCoV-2, in late 2019 and the subsequent global pandemic of COVID19 disease. Despite being asymptomatic or causing only a mild illness in around $80 \%$ of cases, infection may lead to severe respiratory distress with associated morbidity and mortality [29]. Many countries have adopted a policy of shielding groups of vulnerable patients such as the elderly or those with significant comorbidities. Remote assessment of these patients in the community would mitigate the need for them to travel to hospital and hopefully reduce potential exposure. Given the continued presence of this virus worldwide and the potential for further outbreaks, ongoing shielding of vulnerable patients will likely be required.

\section{Future work}

The studies included in this review have demonstrated the potential for video-otoscopy to enhance otological care in various circumstances; however, there is scope for further research. The large number of devices used assessors and training methodology and heterogenous study methodology make it challenging to draw firm conclusions. An emphasis should be placed on a need to compare remote assessment with a 'gold-standard' where possible, as this validates remote assessment as a clinically justifiable option. However, in pragmatic terms, a suboptimal assessment may well be better than no assessment at all. To date, many studies have examined remote or rural populations, however, it would also be pertinent to look at the application of this technology to developed healthcare systems to optimise referrals between primary and secondary care, potentially reducing unneeded referrals and speeding up care for those with identified pathology. Clear synergies exist with the use of adjuvant investigations, such as boothless audiometry and tympanometry, to see if this multimodality approach improves the reliability of remote assessment. The majority of included studies have used a video-otoscope-based system; however, further studies investigating the use of otoendoscopes would be helpful to evaluate the feasibility of non-otolaryngology-specialist facilitators being trained to use them safely and whether the wide-angled view and improved definition leads to the acquisition of higher quality images that are more easily assessed remotely.

\section{Conclusion}

Remote otological assessment using video-otoscopy shows potential as a safe and effective method for detecting the presence of ear disease in a wide range of healthcare settings. Despite this, there are barriers to the acquisition of adequate images, most notably the presence of obstructing cerumen. Strategies to manage this are vital to reduce the failure rate of remote assessment. Likewise, adequate training of facilitators is paramount to maintaining patient safety and maximising the chance of adequate image acquisition. In real-world terms, the ability to identify a 'normal' ear may be more important than the diagnosis of specific pathology, as a binary outcome of 'normal' or 'abnormal' could trigger referral for specialist review. Existing studies have primarily focussed on the use of video-otoscopy, but otoendoscopy may provide improved visualisation. Further work is required to evaluate endoscope-based systems to assess safety of examination by trained facilitators and whether they will allow the acquisition of higher quality images that allow a more comprehensive remote assessment. The usefulness of remotely acquired images may be enhanced by triangulation with boothless audiometry and tympanometry (Fig. 1). 


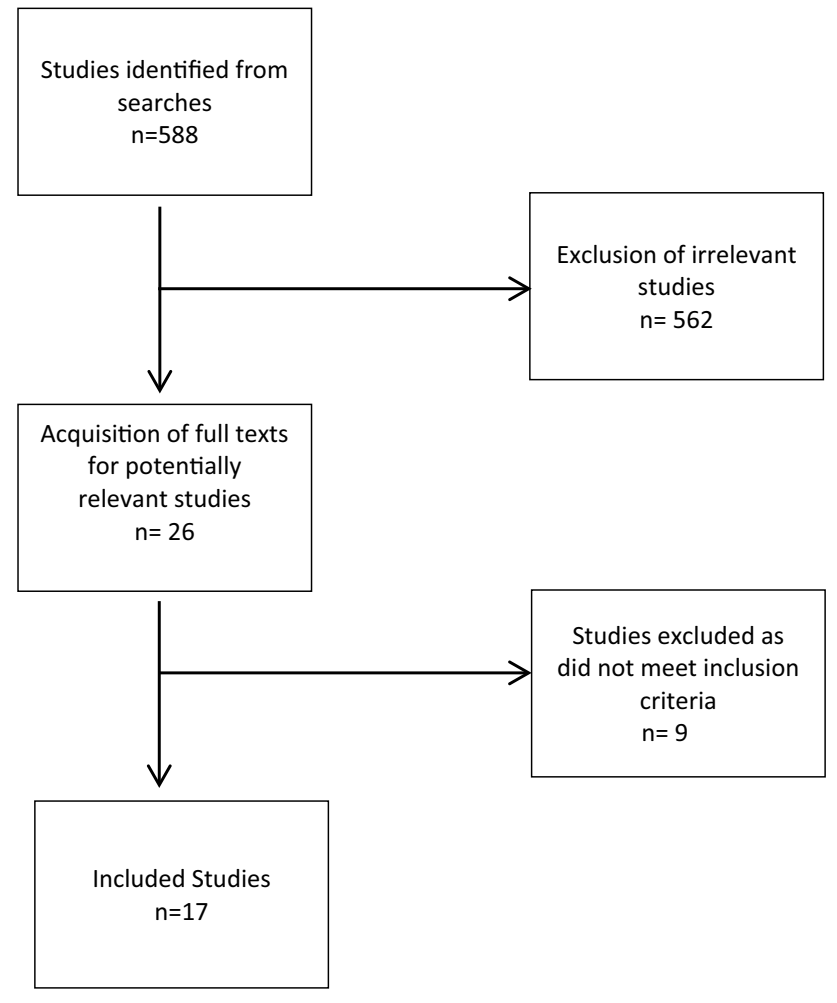

Fig. 1 Summary of search process

Funding None.

Data availability Not applicable.

Code availability Not applicable.

\section{Compliance with ethical standards}

Conflict of interest Mr Chris Coulson is the CEO of endoscope-I Ltd, a developer of physical adaptors and smartphone applications for mobile endoscopic imaging. Mr Muzaffar, Lt Col Orr and Mr Coulson hold grants from UK Defence for the investigation of innovative technology applied to remote assessment of hearing.

Ethics approval Not applicable.

Consent to participate Not applicable.

Consent for publication Not applicable.

\section{References}

1. World Health Organisation (WHO) Deafness and hearing loss. https://www.who.int/news-room/fact-sheets/detail/deafness-andhearing-loss. Accessed 30 Dec 2019

2. Gupta N, Chawla N, Gupta D et al (2017) Community triage of otology patients using a store-and-forward telemedicine device: A feasibility study. Ear Nose Throat J 96:246-249
3. Mandavia R, Lapa T, Smith M, Bhutta MF (2018) A cross-sectional evaluation of the validity of a smartphone otoscopy device in screening for ear disease in Nepal. Clin Otolaryngol 43:31-38. https://doi.org/10.1111/coa.12898

4. NHS England (2019) Transforming elective care services ear, nose and throat (ENT)

5. Moher D, Liberati A, Tetzlaff J et al (2009) Preferred reporting items for systematic reviews and meta-analyses: the PRISMA statement. Ann Intern Med 151(264-269):W64

6. Biagio L, Swanepoel DW, Adeyemo A et al (2013) Asynchronous video-otoscopy with a telehealth facilitator. Telemed J E-Health Off J Am Telemed Assoc 19:252-258. https://doi.org/10.1089/ tmj.2012.0161

7. Damery L, Lescanne E, Reffet K et al (2019) Interest of video-otoscopy for the general practitioner. Eur Ann Otorhinolaryngol Head Neck Dis 136:13-17. https://doi.org/10.1016/j.anorl.2018.10.016

8. Moberly AC, Zhang M, Yu L et al (2018) Digital otoscopy versus microscopy: How correct and confident are ear experts in their diagnoses? J Telemed Telecare 24:453-459. https://doi. org/10.1177/1357633X17708531

9. Sebothoma B, Khoza-Shangase K (2018) A comparison between video otoscopy and standard tympanometry findings in adults living with human immunodeficiency virus (HIV) in South Africa. South Afr J Commun Disord Suid-Afr Tydskr Vir Kommun 65:e1-e7. https://doi.org/10.4102/sajcd.v65i1.591

10. Seim NB, Philips RHW, Matrka LA et al (2018) Developing a synchronous otolaryngology telemedicine Clinic: Prospective study to assess fidelity and diagnostic concordance. The Laryngoscope 128:1068-1074. https://doi.org/10.1002/lary.26929

11. Yulzari R, Bretler S, Avraham Y et al (2018) Mobile technologybased real-time teleotolaryngology care facilitated by a nonotolaryngologist physician in an adult population. Ann Otol Rhinol Laryngol 127:46-50. https://doi.org/10.1177/000348941774508 9

12. Lundberg T, Biagio L, Laurent $C$ et al (2014) Remote evaluation of video-otoscopy recordings in an unselected pediatric population with an otitis media scale. Int J Pediatr Otorhinolaryngol 78:1489-1495. https://doi.org/10.1016/j.ijporl.2014.06.018

13. Lundberg T, Biagio de Jager L, Swanepoel DW, Laurent C (2017) Diagnostic accuracy of a general practitioner with video-otoscopy collected by a health care facilitator compared to traditional otoscopy. Int J Pediatr Otorhinolaryngol 99:49-53. https://doi. org/10.1016/j.ijporl.2017.04.045

14. Biagio L, Swanepoel DW, Laurent C, Lundberg T (2014) Videootoscopy recordings for diagnosis of childhood ear disease using telehealth at primary health care level. J Telemed Telecare 20:300-306. https://doi.org/10.1177/1357633X14541038

15. Demant MN, Jensen RG, Bhutta MF et al (2019) Smartphone otoscopy by non-specialist health workers in rural Greenland: a cross-sectional study. Int J Pediatr Otorhinolaryngol 126:109628. https://doi.org/10.1016/j.ijporl.2019.109628

16. Erkkola-Anttinen N, Irjala H, Laine MK et al (2019) Smartphone otoscopy performed by parents. Telemed J E-Health Off J Am Telemed Assoc 25:477-484. https://doi.org/10.1089/tmj.2018.0062

17. Shah MU, Sohal M, Valdez TA, Grindle CR (2018) iPhone otoscopes: currently available, but reliable for tele-otoscopy in the hands of parents? Int J Pediatr Otorhinolaryngol 106:59-63. https ://doi.org/10.1016/j.ijporl.2018.01.003

18. Yancey KL, Cheromei LJ, Muhando J et al (2019) Pediatric hearing screening in low-resource settings: incorporation of video-otoscopy and an electronic medical record. Int J Pediatr Otorhinolaryngol 126:109633. https://doi.org/10.1016/j.ijpor 1.2019 .109633

19. Ramkumar V, Rajendran A, Nagarajan R et al (2018) Identification and management of middle ear disorders in a rural cleft care 
program: a telemedicine approach. Am J Audiol 27:455-461. https://doi.org/10.1044/2018_AJA-IMIA3-18-0015

20. Wu C-J, Wu S-Y, Chen P-C, Lin Y-S (2014) An innovative smartphone-based otorhinoendoscope and its application in mobile health and teleotolaryngology. J Med Internet Res 16:e71. https ://doi.org/10.2196/jmir.2959

21. Burton MJ, Dorée CJ (2003) Ear drops for the removal of ear wax. Cochrane Database Syst Rev CD004400. https://doi. org/10.1002/14651858.CD004400

22. Memel D, Langley C, Watkins C et al (2002) Effectiveness of ear syringing in general practice: a randomised controlled trial and patients' experiences. Br J Gen Pract 52:906-911

23. Jones WS, Kaleida PH, Lopreiato JO (2004) Assessment of pediatric residents' otoscopic interpretive skills by videotaped examinations. Ambul Pediatr Off J Ambul Pediatr Assoc 4:162-165. https://doi.org/10.1367/A03-017R1.1

24. Eikelboom RH, Weber S, Atlas MD et al (2003) A tele-otology course for primary care providers. J Telemed Telecare 9(Suppl 2):S19-22. https://doi.org/10.1258/135763303322596156

25. Jacups SP, Newman D, Dean D et al (2017) An innovative approach to improve ear, nose and throat surgical access for remote living Cape York Indigenous children. Int J Pediatr
Otorhinolaryngol 100:225-231. https://doi.org/10.1016/j.ijpor 1.2017.07.011

26. Koch A, Homøe P, Pipper C et al (2011) Chronic suppurative otitis media in a birth cohort of children in Greenland: population-based study of incidence and risk factors. Pediatr Infect Dis J 30:25-29. https://doi.org/10.1097/INF.0b013e3181efaa11

27. Morris PS (1998) A systematic review of clinical research addressing the prevalence, aetiology, diagnosis, prognosis and therapy of otitis media in Australian Aboriginal children. J Paediatr Child Health 34:487-497. https://doi.org/10.104 6/j.1440-1754.1998.00299.x

28. World Health Organization (WHO). (2004) Chronic suppurative otitis media: Burden of illness and management options

29. Wu Z, McGoogan JM (2020) Characteristics of and important lessons from the Coronavirus disease 2019 (COVID-19) Outbreak in China: summary of a report of 72314 cases from the Chinese Center for disease control and prevention. JAMA. https://doi. org/10.1001/jama.2020.2648

Publisher's Note Springer Nature remains neutral with regard to jurisdictional claims in published maps and institutional affiliations. 